\title{
Long Chain Dicationic Phase Transfer Catalysts in the Condensation Reactions of Aromatic Aldehydes in Water Under Ultrasonic Effect
}

\author{
Ilker Esen, Cigdem Yolacan, and Feray Aydogan* \\ Department of Chemistry, Yildiz Technical University, Davutpasa Campus, 34010, Esenler, Istanbul, Turkey \\ *E-mail: feray_aydogan@yahoo.com \\ Received May 15, 2010, Accepted June 23, 2010
}

\begin{abstract}
Long chain dicationic ammonium salts were used successfully as phase transfer catalyst in the condensation reactions of aromatic aldehydes in water under ultrasonic irradiation for the first time. The quaternary salt having longer distance between the cation centers was more effective than the mono- and dicationic ones having short chain.
\end{abstract}

Key Words: Phase transfer catalyst, Ultrasound-assisted synthesis, Aromatic aldehyde, Arylacrylonitrile, $\alpha, \beta$ Unsaturated ketone

\section{Introduction}

The development of new synthetic methods that are more environmentally benign has been propelled by the growing importance of green chemistry. ${ }^{1}$ In this fact, scientists have been aimed to produce safer and environmentally friendly chemicals and processes in recent years. Among these processes, using catalytic reactions is essential for chemists. ${ }^{2}$ Phase transfer catalysis (PTC) is an alternative solution to heterogeneity problem with the reactions in which the interaction between two substances located in different phases of a mixture is inhibited because of the inability of reagents to come together. The most important advantages of PTC are simplicity, mild conditions, high-reaction rates, high selectivities, and the use of inexpensive reagents. ${ }^{3}$ A number of catalysts are being developed and recently multi-site phase transfer catalysts have become more attractive among chemists because of their superior features such as facile preparation, low energy requirement and high reactivity in a particular synthetic transformation under mild reaction conditions, when compared with single-site ones. ${ }^{4}$

Dicationic ammonium salts, which have long aliphatic chain, have excellent surfactant properties, because they are more efficient in lowering surface tension than single chain ones and they are very attractive for adsorption applications, analytical separations, solubilization processes. ${ }^{5}$ Although these compounds are widely used as effective emulsifiers, dispersing and anti-foaming agents, they have not been investigated widely for their phase transfer properties. Here we wish to report the use of dicationic ammonium salts having long aliphatic chain as phase transfer catalysts in the condensation reactions of various aromatic aldehydes with benzyl cyanide and 3,3-dimethylbutan2-one in water under ultrasonic effect.

\section{Experimental}

General. All reagents were of commercial quality and reagent quality solvents were used without further purification. IR spectra were determined on a Perkin Elmer, Spectrum One FT-IR spectrometer. NMR spectra were recorded on Mercury VX-400 MHz and Varian Unity-Inova $500 \mathrm{MHz}$ spectrometers.
Chemical shifts $\delta$ are reported in ppm relative to $\mathrm{CHCl}_{3}\left({ }^{1} \mathrm{H}\right.$ : $\delta=7.27), \mathrm{CDCl}_{3}\left({ }^{13} \mathrm{C}: \delta=77.0\right)$ and TMS as internal standart. Column chromatography was conducted on silica gel 60 (70 230 mesh). TLC was carried out on aluminum sheets precoated with silica gel $60 \mathrm{~F}_{254}$ (Merck). Elemental analysis were carried out on Thermo Flash EA 1112 series apparatus. Ultrasound irradiation was performed in Bandelin Sonorex ultrasonic cleaner, whose frequency was $35 \mathrm{kHz}$ and output power was $350 \mathrm{~W}$. The reaction flasks were located in the maximum energy area in the cleaner, and the temperature of the water bath was controlled by the circulation of cold water by a pump.

General procedure for the synthesis of catalysts 1 a-c. A mixture of $N, N$-dimethyldodecylamine $(2.0 \mathrm{mmol})$ and dihaloalkane $(1.0 \mathrm{mmol})$ in absolute ethanol $(10 \mathrm{~mL})$ was refluxed for $24 \mathrm{~h}$. After evaporation of the solvent, the crude product was washed with acetone and recrystallized from ethanol/diethyl ether (1:2).

1,3-Bis(dodecyldimethylamino)propane dibromide (1a): White powder. Yield: $597 \mathrm{mg}, 95 \%$, mp 118 - $119^{\circ} \mathrm{C}$. IR (atr) $v 2948,2916,2870,2849,1486,1471,1463,1433,1382 \mathrm{~cm}^{-1}$; ${ }^{1} \mathrm{H} \mathrm{NMR}\left(400 \mathrm{MHz}, \mathrm{CDCl}_{3}\right) \delta 0.85\left(\mathrm{t}, J=5.4 \mathrm{~Hz}, 6 \mathrm{H}, \mathrm{CH}_{3}\right), 1.23$ (brs, 22H, $\mathrm{CH}_{2}$ ), 1.32 (brs, 8H, $\mathrm{CH}_{2}$ ), 1.72 (brs, 4H, $\mathrm{CH}_{2}$ ), 2.07 (brs, $4 \mathrm{H}, \mathrm{CH}_{2}$ ), 2.85 (brs, $4 \mathrm{H}, \mathrm{CH}_{2}$ ), 3.27 (s, 12H, N-CH $), 3.40$ (m, 4H, $\mathrm{CH}_{2}$ ), 3.81 (brs, $4 \mathrm{H}, \mathrm{CH}_{2}$ ); Anal. Calcd for $\mathrm{C}_{31} \mathrm{H}_{68} \mathrm{Br}_{2} \mathrm{~N}_{2}$ (628.69): C, 59.22; H, 10.90; N, 4.46. Found: C, 59.15; H, 10.87; $\mathrm{N}, 4.55$.

1,6-Bis(dodecyldimethylamino)hexane dibromide (1b): White powder. Yield: $657 \mathrm{mg}, 98 \%, \mathrm{mp} 225-226^{\circ} \mathrm{C}$. IR (atr) v 2949, 2916, 2850, 1484, 1464, 1401, $1376 \mathrm{~cm}^{-1}$; ${ }^{1} \mathrm{H}$ NMR $\left(400 \mathrm{MHz}, \mathrm{CDCl}_{3}\right) \delta 0.82\left(\mathrm{t}, J=6.8 \mathrm{~Hz}, 6 \mathrm{H}, \mathrm{CH}_{3}\right), 1.22(\mathrm{~m}$, $28 \mathrm{H}, \mathrm{CH}_{2}$ ), 1.29 (brs, 8H, $\mathrm{CH}_{2}$ ), 1.51 (brs, $4 \mathrm{H}, \mathrm{CH}_{2}$ ), 1.66 (brs, $\left.4 \mathrm{H}, \mathrm{CH}_{2}\right), 1.93$ (brs, 4H, $\left.\mathrm{CH}_{2}\right), 3.33$ (s, 12H, N-CH $), 3.44$ (m, $\left.4 \mathrm{H}, \mathrm{CH}_{2}\right), 3.64\left(\mathrm{~m}, 4 \mathrm{H}, \mathrm{CH}_{2}\right)$; Anal. Calcd for $\mathrm{C}_{34} \mathrm{H}_{74} \mathrm{Br}_{2} \mathrm{~N}_{2}$ (670.77): C, 60.88; H, 11.12; N, 4.18. Found: C, 60.79; H, 11.08; $\mathrm{N}, 4.23$.

1,12-Bis(dodecyldimethylamino)dodecane dibromide (1c): White powder. Yield: $732 \mathrm{mg}, 97 \%$, mp $135-136{ }^{\circ} \mathrm{C}$ (Lit. ${ }^{5 e}$ $129-134^{\circ} \mathrm{C}$ ). IR (atr) v 2955, 2916, 2851, 1488, 1469, 1421, $1400,1378 \mathrm{~cm}^{-1} ;{ }^{1} \mathrm{H}$ NMR (400 MHz, $\left.\mathrm{CDCl}_{3}\right) \delta 0.82(\mathrm{t}, J=6.8$ $\left.\mathrm{Hz}, 6 \mathrm{H}, \mathrm{CH}_{3}\right), 1.21$ (m, 40H, $\mathrm{CH}_{2}$ ), 1.32 (brs, 8H, $\mathrm{CH}_{2}$ ), 1.66 (brs, $8 \mathrm{H}, \mathrm{CH}_{2}$ ), 2.43 (brs, $4 \mathrm{H}, \mathrm{CH}_{2}$ ), 3.29 (s, 12H, N-CH $), 3.42$ 
(m, 4H, $\mathrm{CH}_{2}$ ), 3.50 (m, 4H, $\mathrm{CH}_{2}$ ); Anal. Calcd for $\mathrm{C}_{40} \mathrm{H}_{86} \mathrm{Br}_{2} \mathrm{~N}_{2}$ (754.93): C, 63.64; H, 11.48; N, 3.71. Found: C, 63.57; H, 11.41; N, 3.79.

General procedure for the condensation reactions. Sodium hydroxide $(1.0 \mathrm{mmol})$ and catalyst $1 \mathrm{c}$ were dissolved in water $(20 \mathrm{~mL})$, then aldehyde $(1.0 \mathrm{mmol})$ and benzyl cyanide or 3,3dimethylbutan-2-one (1.0 mmol) were added. The reaction mixture was sonicated at $25^{\circ} \mathrm{C}$ for a period of time long enough to complete the reaction (TLC), then the solid formed in the reaction mixture was filtrated, and the crude products were purified by recrystallisation (ethanol) or column chromatography over silica gel (EtOAc:Hexane 1:3 or 1:6).

(Z)-2,3-Diphenylacrylonitrile (4a): White crystals. Yield: $203 \mathrm{mg}, 99 \%$, mp $87-88^{\circ} \mathrm{C}$ (Lit. ${ }^{6} 86-87^{\circ} \mathrm{C}$ ). IR (atr) v 3098, 3053, 3032, 2946, 2218, 1608, $1595 \mathrm{~cm}^{-1}$; ${ }^{1} \mathrm{H}$ NMR (400 MHz, $\left.\mathrm{CDCl}_{3}\right) \delta 7.43(\mathrm{~m}, 6 \mathrm{H}, \mathrm{ArH}), 7.54$ (brs, $\left.1 \mathrm{H},=\mathrm{CH}\right), 7.68(\mathrm{~m}, 2 \mathrm{H}$, $\mathrm{ArH}), 7.88$ (m, 2H, ArH); Anal. Calcd for $\mathrm{C}_{15} \mathrm{H}_{11} \mathrm{~N}$ (205.25): C, 87.77; H, 5.40; N, 6.82. Found: C, 87.69; H, 5.36; N, 6.90.

(Z)-3-(4-Fluorophenyl)-2-phenylacrylonitrile (4b): White crystals. Yield: $221 \mathrm{mg}, 99 \%$, mp $115-116^{\circ} \mathrm{C}$. IR (atr) v 3059, 2962, 2212, 1601, 1593, $1584 \mathrm{~cm}^{-1}$; ${ }^{1} \mathrm{HNMR}\left(500 \mathrm{MHz}, \mathrm{CDCl}_{3}\right)$ $\delta 7.07$ (m, 2H, ArH), 7.33 (m, 3H, ArH), 7.40 (brs, 1H, =CH), 7.57 (m, 2H, ArH), 7.81 (m, 2H, ArH); Anal. Calcd for $\mathrm{C}_{15} \mathrm{H}_{10} \mathrm{FN}$ (223.25): C, 80.70; H, 4.51; N, 6.27. Found: C, 80.66; H, 4.47; $\mathrm{N}, 6.34$.

(Z)-2-Phenyl-3-p-tolylacrylonitrile (4c): White crystals. Yield: $120 \mathrm{mg}, 55 \%, \mathrm{mp} 61-62^{\circ} \mathrm{C}$. IR (atr) v 3030, 2213, 1604, $1590 \mathrm{~cm}^{-1} ;{ }^{1} \mathrm{H}$ NMR $\left(400 \mathrm{MHz}, \mathrm{CDCl}_{3}\right) \delta 2.41\left(\mathrm{~s}, 3 \mathrm{H}, \mathrm{CH}_{3}\right)$, 7.27 (m, 2H, ArH), 7.41 (m, 3H, ArH), 7.50 (brs, 1H, =CH), 7.67 (m, 2H, ArH), 7.80 (m, 2H, ArH); Anal. Calcd for $\mathrm{C}_{16} \mathrm{H}_{13} \mathrm{~N}$ (219.28): C, 87.64; H, 5.98; N, 6.39. Found: C, 87.53; H, 5.86; $\mathrm{N}, 6.42$.

(Z)-3-(4-Methoxyphenyl)-2-phenylacrylonitrile (4d): Yellow crystals. Yield: $158 \mathrm{mg}, 67 \%$, mp $93-94{ }^{\circ} \mathrm{C}$ (Lit. ${ }^{7} 95{ }^{\circ} \mathrm{C}$ ). IR (atr) v 3014, 2965, 2843, 2208, 1602, $1592 \mathrm{~cm}^{-1} ;{ }^{1} \mathrm{H}$ NMR $\left(400 \mathrm{MHz}, \mathrm{CDCl}_{3}\right) \delta 3.87\left(\mathrm{~s}, 3 \mathrm{H}, \mathrm{OCH}_{3}\right), 6.98(\mathrm{~m}, 2 \mathrm{H}, \mathrm{ArH})$, 7.39 (m, 3H, ArH), 7.46 (brs, 1H, =CH), 7.65 (m, 2H, ArH), 7.88 (m, 2H, ArH); Anal. Calcd for $\mathrm{C}_{16} \mathrm{H}_{13} \mathrm{NO}$ (235.28): $\mathrm{C}$, 81.68; H, 5.57; N, 5.95. Found: C, 81.57; H, 5.52; N, 6.02.

(Z)-2-Phenyl-3-(thiophen-2-yl)acrylonitrile (4e): Yellow crystals. Yield: $190 \mathrm{mg}, 90 \%$, mp $96-97{ }^{\circ} \mathrm{C}$. IR (atr) v 3094, $3070,3027,2206,1587 \mathrm{~cm}^{-1} ;{ }^{1} \mathrm{H}$ NMR $\left(400 \mathrm{MHz}, \mathrm{CDCl}_{3}\right) \delta$ 7.14 (m, $1 \mathrm{H}$, thiophen), 7.37 (brd, $J=7.0 \mathrm{~Hz}, 1 \mathrm{H}$, thiophen), 7.43 (m, 2H, ArH), 7.54 (brd, $J=4.6 \mathrm{~Hz}, 1 \mathrm{H}$, thiophen), 7.63 (brs, $1 \mathrm{H},=\mathrm{CH}), 7.65(\mathrm{~m}, 3 \mathrm{H}, \mathrm{ArH})$; Anal. Calcd for $\mathrm{C}_{13} \mathrm{H}_{9} \mathrm{NS}$ (211.28): C, 73.90; H, 4.29; N, 6.63; S, 15.18. Found: C, 73.87; $\mathrm{H}, 4.26 ; \mathrm{N}, 6.68 ; \mathrm{S}, 15.21$.

(Z)-3-(5-Methylfuran-2-yl)-2-phenylacrylonitrile (4f): Light yellow crystals. Yield: $199 \mathrm{mg}, 95 \%, \mathrm{mp} 83-84^{\circ} \mathrm{C}$. IR (atr) $v$ 3056, 3022, 2213, 1615, $1567 \mathrm{~cm}^{-1}$; ${ }^{1} \mathrm{HNMR}$ (400 MHz, $\mathrm{CDCl}_{3}$ ) $\delta 2.41\left(\mathrm{~s}, 3 \mathrm{H}, \mathrm{CH}_{3}\right), 6.19$ (d, $J=3.5 \mathrm{~Hz}, 1 \mathrm{H}$, furan), 7.09 (d, $J=$ $3.5 \mathrm{~Hz}, 1 \mathrm{H}$, furan), 7.30 (brs, $1 \mathrm{H},=\mathrm{CH}), 7.38(\mathrm{~m}, 3 \mathrm{H}, \mathrm{ArH})$, $7.61(\mathrm{~m}, 2 \mathrm{H}, \mathrm{ArH}) ;{ }^{13} \mathrm{C} \mathrm{NMR}\left(100 \mathrm{MHz}, \mathrm{CDCl}_{3}\right) \delta 14,106,110$, $117,119,126,128,129,130,135,149,156$; Anal. Calcd for $\mathrm{C}_{14} \mathrm{H}_{11} \mathrm{NO}$ (209.24): C, 80.36; H, 5.30; N, 6.69. Found: C, 80.31; $\mathrm{H}, 5.23 ; \mathrm{N}, 6.72$.

(E)-4,4-Dimethyl-1-phenylpent-1-en-3-one (5a): White crystals. Yield: $186 \mathrm{mg}, 99 \%$, mp $41-42{ }^{\circ} \mathrm{C}$ (Lit. ${ }^{8} 38-40{ }^{\circ} \mathrm{C}$ ). IR (atr) v 3061, 3026, 2967, 2868, 1682, 1608, 1576, $1476 \mathrm{~cm}^{-1}$; ${ }^{1} \mathrm{H}$ NMR $\left(400 \mathrm{MHz}, \mathrm{CDCl}_{3}\right) \delta 1.23\left(\mathrm{~s}, 9 \mathrm{H}, \mathrm{CH}_{3}\right), 7.12(\mathrm{~d}, J=$ $15.6 \mathrm{~Hz}, 1 \mathrm{H},=\mathrm{CH}), 7.37$ (m, 3H, ArH), 7.56 (m, 2H, ArH), $7.68(\mathrm{~d}, J=15.6 \mathrm{~Hz}, 1 \mathrm{H},=\mathrm{CH})$; Anal. Calcd for $\mathrm{C}_{13} \mathrm{H}_{16} \mathrm{O}$ (188.27): C, 82.94; H, 8.57. Found: C, 82.89; H, 8.52.

(E)-1-(4-Fluorophenyl)-4,4-dimethylpent-1-en-3-one (5b): White crystals. Yield: $196 \mathrm{mg}, 95 \%, \mathrm{mp} 50-51{ }^{\circ} \mathrm{C}$. IR (atr) $v$ 3043, 2968, 2934, 2868, 1675, 1611, 1596, $1475 \mathrm{~cm}^{-1}$; ${ }^{1} \mathrm{H}$ NMR $\left(400 \mathrm{MHz}, \mathrm{CDCl}_{3}\right) \delta 1.15\left(\mathrm{~s}, 9 \mathrm{H}, \mathrm{CH}_{3}\right), 6.98(\mathrm{~m}, 3 \mathrm{H}, \mathrm{ArH}$ and $=\mathrm{CH}), 7.48$ (m, 2H, ArH), $7.56(\mathrm{~d}, J=15.6 \mathrm{~Hz}, 1 \mathrm{H},=\mathrm{CH})$; Anal. Calcd for $\mathrm{C}_{13} \mathrm{H}_{15} \mathrm{FO}(206.26)$ : C, 75.70; H, 7.33. Found: C, 75.67; H, 7.28.

(E)-4,4-Dimethyl-1-p-tolylpent-1-en-3-one (5c): Yellow crystals. Yield: $81 \mathrm{mg}, 40 \%$, mp $79-80{ }^{\circ} \mathrm{C}$. IR (atr) v 3028, 2959, 2926, 2867, 1673, 1600, 1567, $1473 \mathrm{~cm}^{-1}$; ${ }^{1} \mathrm{H}$ NMR (400 MHz, $\left.\mathrm{CDCl}_{3}\right) \delta 1.15\left(\mathrm{~s}, 9 \mathrm{H}, \mathrm{CH}_{3}\right), 2.30\left(\mathrm{~s}, 3 \mathrm{H}, \mathrm{CH}_{3}\right), 7.01(\mathrm{~d}, J=15.6$ $\mathrm{Hz}, 1 \mathrm{H},=\mathrm{CH}), 7.12(\mathrm{~d}, J=8.2 \mathrm{~Hz}, 2 \mathrm{H}, \mathrm{ArH}), 7.40(\mathrm{~d}, J=8.2$ $\mathrm{Hz}, 2 \mathrm{H}, \mathrm{ArH}), 7.59(\mathrm{~d}, J=15.6 \mathrm{~Hz}, 1 \mathrm{H},=\mathrm{CH})$; Anal. Calcd for $\mathrm{C}_{14} \mathrm{H}_{18} \mathrm{O}$ (202.29): C, 83.12; H, 8.97. Found: C, 83.06; H, 8.92.

(E)-4,4-Dimethyl-1-(thiophen-2-yl)pent-1-en-3-one (5e): Yellow oil. Yield: $180 \mathrm{mg}, 93 \%$. IR (atr) v 3106, 3078, 2965, 2930, 2867, 1676, 1587, $1475 \mathrm{~cm}^{-1}$; ${ }^{1} \mathrm{HNMR}\left(400 \mathrm{MHz}, \mathrm{CDCl}_{3}\right)$ $\delta 1.14\left(\mathrm{~s}, 9 \mathrm{H}, \mathrm{CH}_{3}\right), 6.83(\mathrm{~d}, J=15.6 \mathrm{~Hz}, 1 \mathrm{H},=\mathrm{CH}), 6.98(\mathrm{dd}$, $J=5.0,3.9 \mathrm{~Hz}, 1 \mathrm{H}$, thiophen), 7.21 (brd, $J=3.9 \mathrm{~Hz}, 1 \mathrm{H}$, thiophen), 7.29 (brd, $J=4.3 \mathrm{~Hz}, 1 \mathrm{H}$, thiophen), 7.71 (d, $J=15.2 \mathrm{~Hz}$, $1 \mathrm{H},=\mathrm{CH}$ ); Anal. Calcd for $\mathrm{C}_{11} \mathrm{H}_{14} \mathrm{OS}$ (194.29): C, 68.00; H, 7.26; S, 16.50. Found: C, 67.94; H, 7.23; S, 16.54.

(E)-4,4-Dimethyl-1-(5-methylfuran-2-yl)pent-1-en-3-one (5f): Yellow oil. Yield: $163 \mathrm{mg}, 85 \%$. IR (atr) v 3113, 2967, 2869, 1676, 1608, 1571, $1476 \mathrm{~cm}^{-1}$; ${ }^{1} \mathrm{H} \mathrm{NMR}\left(400 \mathrm{MHz}, \mathrm{CDCl}_{3}\right)$ $\delta 1.14\left(\mathrm{~s}, 9 \mathrm{H}, \mathrm{CH}_{3}\right), 2.29\left(\mathrm{~s}, 3 \mathrm{H}, \mathrm{CH}_{3}\right), 6.05(\mathrm{dd}, J=3.5,1.2$ $\mathrm{Hz}, 1 \mathrm{H}$, furan), 6.46 (d, $J=3.5 \mathrm{~Hz}, 1 \mathrm{H}$, furan), 6.84 (d, $J=15.2$ $\mathrm{Hz}, 1 \mathrm{H},=\mathrm{CH}), 7.29(\mathrm{~d}, J=15.2 \mathrm{~Hz}, 1 \mathrm{H},=\mathrm{CH})$; Anal. Calcd for $\mathrm{C}_{12} \mathrm{H}_{16} \mathrm{O}_{2}$ (192.25): C, 74.97; H, 8.39. Found: C, 74.91; H, 8.33.

\section{Results and Discussion}

The ammonium salts having different spacer lengths were synthesized by the reaction of dibromoalkanes with $N, N$-dimethyldodecylamine according to literature procedure (Scheme 1). The structures of compounds were determined by their spectroscopic data and were in accordance with the literature. ${ }^{5 b}$

We started the study by investigation of the condensation reaction of benzyl cyanide with $p$-fluorobenzaldehyde to see how the long chain aliphatic dicationic ammonium salts effected the reaction as phase transfer catalysts. The reactions were carried out in water-reagent/substrate biphasic system without any other organic solvent. Several different bases such as sodium hydroxide, triethylamine, potassium carbonate were tried as base in the presence of catalysts $\mathbf{1 a - c}$ and it was decided that

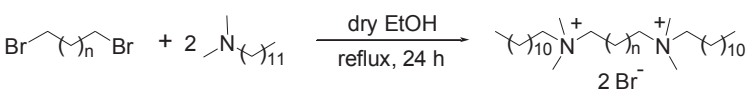

$$
\begin{aligned}
& \text { 1a, } n=1 \\
& \text { 1b, } n=4 \\
& \text { 1c, } n=10
\end{aligned}
$$

Scheme 1. The synthesis of dicationic phase transfer catalysts 
Table 1. The comparison of the activity of catalysts

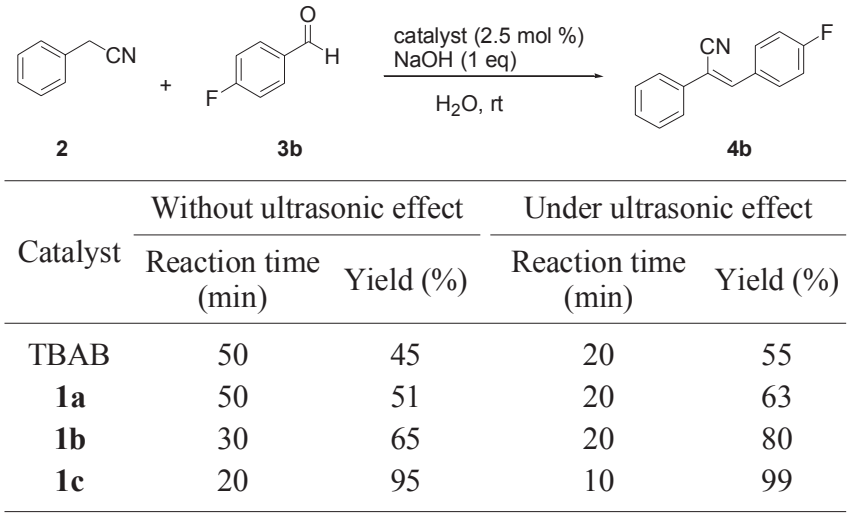

Table 2. The synthesis of acrylonitrile derivatives

Reaction time $\begin{gathered}\text { Yield } \\ \text { (min) }\end{gathered}$

sodium hydroxide was the good base in one equimolar amount. Then different catalyst amounts such as 10, 5, 2.5, and 1\% were tried and the obtained yields were $95,94,95$, and $57 \%$, respectively for the catalyst $\mathbf{1 c}$ as an example, so $2.5 \% \mathrm{~mol}$ catalyst was the best amount. Under these optimum conditions, the reaction was carried out with catalysts 1a-c and also tetrabutylammonium bromide (TBAB) which is monocationic short chain ammonium salt at room temperature with or without ultrasonic irradiation for comparison. As one can see from the yields given in the Table 1, the catalyst $\mathbf{1 c}$ having longest aliphatic
Table 3. The synthesis of 1-aryl-4,4-dimethylpent-1-en-3-one compounds

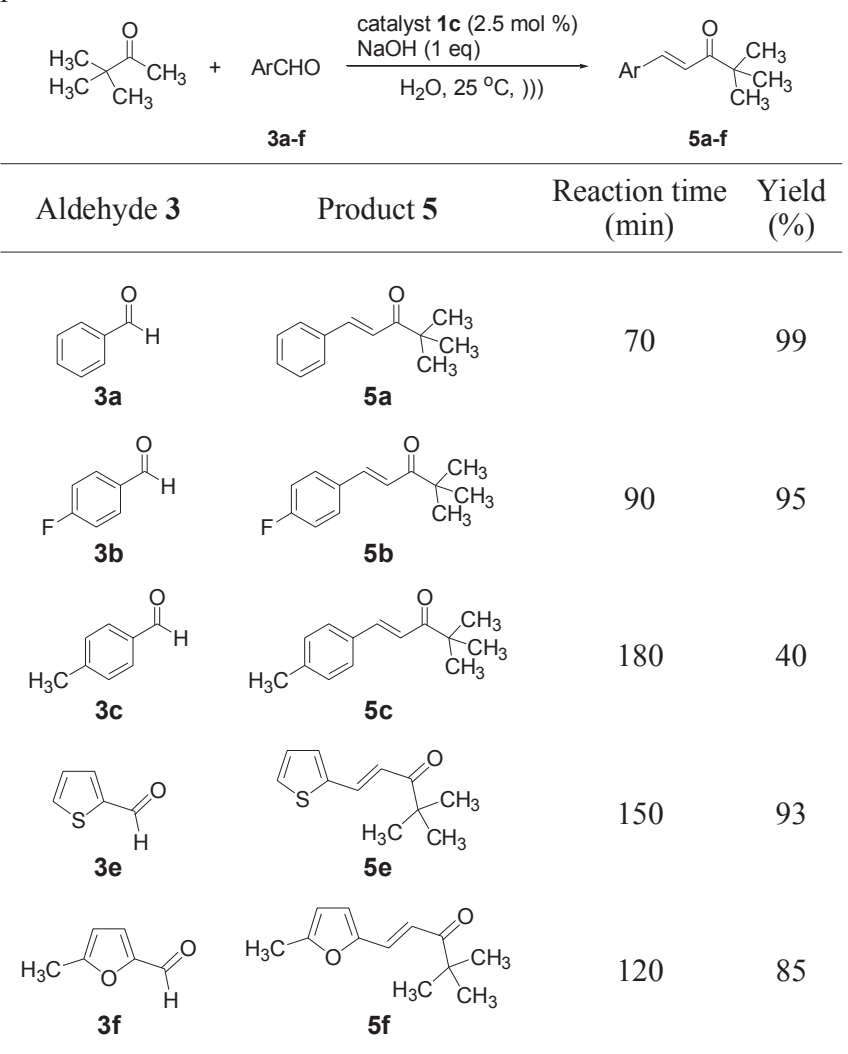

chain was the most effective one. This can be interpreted as the catalyst cation having long alkyl group is easily dispersed into the organic substrate phase because of the lipophilicity of the cation. Beside, it is possible to think that the cationic centers separated by short aliphatic chain may interact with the anion more tightly than those separated by long chain, and exhibit a lower activity in activating anions by preventing exchange the anions for the reaction. So the electronic factors might not be important with the quaternary salts having longer distances between the cation centers, and these salts exhibit higher activity in activating anions. 9

Using this procedure, the condensation reactions of various aromatic aldehydes with benzyl cyanide and 3,3-dimethylbutan2-one were carried out in good yields and short reaction times as summarised in Table 2 and 3. All of the compounds except $4 \mathbf{f}$ were known in the literature and all the spectroscopic data are in full agreement with their structures. Under ultrasonic irradiation, all hydroxy compounds, which formed according to the reaction mechanism were converted to unsaturated compounds immediately by the elimination of water, so no hydroxy product was isolated.

\section{Conclusion}

Long chain dicationic ammonium salts, which are prepared easily from cheap, commercially available materials, are very effective as phase transfer catalysts in the condensation reactions of aromatic aldehydes. When the aliphatic chain between the cationic centers is longer, the phase transfer activity in- 
creases. Besides, the method used for the condensation reactions provides several advantages such as using environmentally friendly solvent water, short reaction times under ultrasonic irradiation and high yielded clean products.

Acknowledgments. We thank Yildiz Technical University Scientific Research Foundation (BAPK Project number 28-0102-02) for financial support.

\section{References}

1. Anastas, P. T.; Warner, J. C. In Green Chemistry: Theory and Practice; Oxford University Press: New York, 1998. (b) Sanghi, R. Curr. Sci. 2000, 79, 1662

2. Sheldon, R. A.; Arends, I.; Hanefeld, U. Green Chemistry and Catalysis, 3rd ed.; Wiley-VCH Verlag: Weinheim, 2008.

3. (a) Starks, C. M.; Liotta, E. L.; Halpern, M. In Phase Transfer Catalysis Fundamentals, Applications and Industrial Perspectives; Chapman and Hall: New York, 1994. (b) Makosza, M. Pure Appl. Chem. 2000, 72, 1399. (c) Jones, R. A. In Quaternary Ammonium Salts Their Use in Phase Transfer Catalysis; Academic Press:
Trowbridge, 2001. (d) Ahmed, B.; Khan, R. A.; Keshari, M. Tetrahedron Lett. 2009, 50, 2889. (e) Han, Z.; Yamaguchi, Y.; Kitamura, M.; Maruoka, K. Tetrahedron Lett. 2005, 46, 8555. (f) O'Reilly, E.; Lestini, E.; Balducci, D.; Paradisi, F. Tetrahedron Lett. 2009, $50,1748$.

4. Vivekanand, P. A.; Balakrishnan, T. Catal. Commun. 2009, 10, 687. (b) Ali, H. S. Catal. Commun. 2007, 8, 855. (c) Murugan, E.; Gopinath, P. J. Mol. Catal. A-Chem. 2009, 309, 12. (d) Park, H.; Jeong, B.; Yoo, M.; Park, M.; Huh, H.; Jew, S. Tetrahedron Lett. 2001, 42, 4645. (e) Murugan, E.; Siva, A. J. Mol. Catal. A-Chem. 2005, 235, 220.

5. Sekhon, B. S. Resonance 2004, 42. (b) Bagha, A. R. T.; Bahrami, H.; Movassagh, B.; Arami, M.; Menger, F. M. Dyes Pigm. 2007, 72, 331. (c) Li, F.; Rosen, M. J. J. Colloid. Interface Sci. 2000, 224, 265. (d) Shukla, D.; Tyagi, V. K. J. Oleo. Sci. 2006, 55, 381. (e) Buwalda, R. T.; Engberts, B. F. N. Langmuir 2001, 17, 1054.

6. Villemin, D.; Jullien, A.; Bar, N. J. Mol. Catal. A-Chem. 2003, $5,467$.

7. Loupy, A.; Pellet, M.; Petit, A.; Vo-Thanh, G. Org. Biomol. Chem. 2005, 3, 1534

8. Peach, P.; Cross, D. J.; Kenny, J. A.; Mann, I.; Houson, I.; Campbell, L.; Walsgroveb, T.; Willsa, M. Tetrahedron 2006, 62, 1864.

9. Jayachandran, J. P.; Wheeler, C.; Eason, B. C.; Liotta, C. L.; Eckert, C. A. J. Supercrit. Fluid 2003, 27, 179. 